\title{
Incidência do canal médio-mesial em primeiros molares inferiores através de
}

\section{Tomografia Computadorizada Cone Beam}

\author{
Analysis of the middle mesial canals incidence of mandibular first molars using Cone Beam \\ computed tomography
}

Incidencia del canal médio-mesial em primeiros molares mandibulares a través de tomografia computadorizada Cone Beam

Luciano Madeira ORCID: https://orcid.org/0000-0002-7991-0538 Universidade da Região de Joinville, Brasil E-mail: madeiraluciano0@gmail.com

Pietra Linzmeyer Werner de Lima ORCID: https://orcid.org/0000-0001-9744-4342 Universidade da Região de Joinville, Brasil E-mail: linzmeyerpietra@gmail.com

Thiago Geronimo

ORCID: https://orcid.org/0000-0001-7693-9880 Universidade da Região de Joinville, Brasil E-mail: geronimothiago@gmail.com

Giuseppe Valduga Cruz ORCID: https://orcid.org/0000-0002-1897-8803 Universidade da Região de Joinville, Brasil E-mail: giuseppevalduga@gmail.com

Flávia Tomazinho

ORCID: https://orcid.org/0000-0001-5553-6943 Universidade Positivo, Brasil E-mail: flavia.tomazinho@gmail.com

Flares Baratto Filho

ORCID: https://orcid.org/0000-0002-5649-7234 Universidade da Região de Joinville, Brasil E-mail: fbaratto1 @gmail.com

\begin{abstract}
Resumo
Introdução: Inúmeros estudos têm se preocupado com a incidência do canal médio-mesial na raiz mesial de primeiros molares inferiores e os dados são bem variados devido a inúmeros fatores relacionados às metodologias dos estudos. Objetivo: Avaliar a incidência do canal médio-mesial em primeiros molares inferiores por meio da análise de imagens tomográficas. Material e métodos: A amostra de 81 imagens tomográficas, 133 primeiros molares inferiores, realizadas com o tomógrafo Orthopantomograph OP300 e o sistema OnDemand 3D Dental, foram observados em corte axial mandibular, quanto à presença do canal médio-mesial e sua configuração anatômica. Resultados: Constatou-se que a incidência do canal médio-mesial foi de $23,30 \%$ do total da amostra. Conforme classificação de Vertucci: $66,6 \%$ tipo II; 3,33\% tipo VI; e, 30\% como tipo VIII. Segundo Versiani et al.: 30\% terminavam em forame independente; $3,33 \%$ quatro condutos independentes; e, $66,6 \%$ com confluência para os canais mesiovestibular ou mesiolingual. Conclusão: A incidência do canal médio-mesial em primeiros molares inferiores foi de $23,30 \%$, por meio da análise tomográfica.
\end{abstract}

Palavras-chave: Tomografia computadorizada de feixe cônico; Endodontia; Dente molar; Canal radicular.

\begin{abstract}
Introduction: Numerous studies have evaluated the incidence of the middle-mesial canal in the mesial root of mandibular first molars. However, the available data is varied due to numerous factors related to study methodologies.Aim: To evaluate the incidence of the middle-mesial canal in mandibular first molars through tomographic image analysis. Methodology: A sample of 81 tomographic images of 133 mandibular first molars (acquired with the Orthopantomograph OP300 and the OnDemand 3D Dental system), were observed in mandibular axial section to investigate the presence of the middle-mesial canal and its anatomical configuration. Results: The incidence rate of the middle-mesial canal was $23.30 \%$ in the included samples. According to Vertucci's classification: 66.6\% type II; 3.33\% type VI; and 30\% type VIII. According to Versiani et al., 30\% ended in an independent
\end{abstract}


foramen, $3.33 \%$ in four independent conduits, and $66.6 \%$ with confluence for the mesiobuccal or mesiolingual canals. Conclusion: The incidence of the middle-mesial canal corresponded to $23.30 \%$ of the total sample as evaluated by tomographic analysis.

Keywords: Cone-Beam computed tomography; Endodontics; Molar teeth; Root canal.

\section{Resumen}

Introducción: Numerosos estudios se han ocupado de la incidencia del canal mdeio-mesial en la raíz mesial de los primeros molares mandibulares. Sin embargo, los datos disponibles son variados debido a numerosos factores relacionados con las metodologías de los estudios. Objetivo: Evaluar la incidencia del canal medio-mesial en los primeros molares mandibulares mediante el análisis de imágenes tomográficas. Material y métodos: Una muestra de 81 imágenes tomográficas de 133 primeros molares mandibulares adquiridas con el Orthopantomograph OP300 y el sistema OnDemand 3D Dental, fueron observadas en sección axial mandibular, cuanto a la presencia del canal mediomesial y su configuración anatómica. Resultados: Se comprobó que la incidencia del canal medio-mesial era del 23,30\% del total de la muestra. Según la clasificación de Vertucci: $66,6 \%$ tipo II; 3,33\% tipo VI; y 30\% como tipo VIII. Según Versiani et al.: el 30\% terminaba en foramen independiente; el 3,33\% en cuatro conductos independientes; y el $66,6 \%$ con confluencia para los canales mesiobucales o mesiolinguales. Conclusiones: La incidencia del canal medio-mesial correspondió al 23,30\% del total de la muestra evaluada por el análisis tomográfico.

Palabras clave: Tomografía computarizada Cone Beam; Endodoncia; Dientes molares; Conducto radicular.

\section{Introdução}

O conhecimento da anatomia do sistema de canais radiculares tem importância fundamental na terapia endodôntica, servindo como parâmetro sob os quais o tratamento endodôntico é realizado, podendo afetar as possibilidades de sucesso (Versiani et al.,2016; Weinberg et al., 2020).

A variação na morfologia dos canais radiculares de dentes permanentes e sua relação com procedimentos endodônticos vem sendo pesquisada ao longo dos anos (Tomaszewska et al., 2018; Versiani et al., 2016; Vertucci, 1984). Dentre os dentes pesquisados, os molares inferiores têm sido alvo de algumas pesquisas que destacaram uma enorme variação anatômica (Aminsobhani et al., 2010; Kuzekanani et al., 2020; de Toubes et al., 2012; Srivastava et al., 2018; Versiani et al.,2016; Weinberg et al., 2020; Xu et al., 2020; Yang et al., 2020) podendo apresentar inúmeras configurações e quantidade de canais radiculares.

As radiografias são essenciais para revelar características anatômicas da raiz do dente e parte da anatomia interna, no entanto, para maior detalhe de informações, como o reconhecimento de um quarto canal na raiz de primeiros molares, apresenta grande limitação. Dessa forma, atualmente a Tomografia Computadorizada de Feixe Cônico (TCFC) é uma realidade clínica que permite a visualização tridimensional da imagem (Patel et al, 2007; Patel et al., 2019). Dentre suas inúmeras aplicações na endodontia, destaca-se estudos da morfologia do canal radicular, sendo possível estabelecer o número, localização, forma, tamanho e direção dos canais radiculares (Cotton et al., 2007; Patel et al, 2007; Patel et al., 2019; Weinberg et al., 2020), informações importantes para o planejamento operatório.

Inúmeros estudos recentes, clínicos e experimentais, bem como relatos de casos clínicos, com uso de microscopia operatória, tomografia computadorizada e Microtomografia (Tomaszewska et al., 2018; Versiani et al.,2016; Weinberg et al., 2020) têm possibilitado o estudo da incidência do canal médio-mesial (MM) em molares inferiores, com divergências quanto ao percentual de incidência reportado, com achados que variaram entre nenhum caso identificado até uma prevalência de 53,84\% (Karapinar-Kazandag et al., 2010; Versiani et al.,2016; Tahmasbi et al., 2017; Srivastava et al., 2018; Tomaszewska et al., 2018; Weinberg et al., 2020).

Com base nessa problemática, o objetivo deste trabalho foi avaliar a incidência do canal médio-mesial em primeiros molares inferiores por meio da tomografia computadorizada de feixe cônico. A hipótese estabelecida foi que a incidência do canal médio-mesial estaria de acordo com os achados na literatura em que foram utilizados tomografia, microtomografia e meios de magnificação. 


\section{Metodologia}

Este projeto de pesquisa foi realizado após aprovação no Comitê de Ética em Pesquisa Institucional, sob parecer CAAE: 06989318.6.0000.5366.

Em um banco de dados de uma clínica radiológica, foram selecionadas, incluídas no estudo, todas as imagens tomográficas de primeiros molares inferiores, unilateral ou bilateral, realizadas por meio do tomógrafo computadorizado de feixe cônico Orthopantomograph ${ }^{\circledR}$ OP300 (InstrumentariumImaging Dental, Milwaukee, EUA) e avaliados no sistema OnDemand3D Dental (Cybermed Inc., Seoul, Republicof South Korea). Foram excluídos do estudo casos em que o paciente utilizava aparelho ortodôntico, especialmente braquetes ou banda, ou apresentavam procedimentos endodônticos e reabilitadores nos dentes avaliados, que pudessem gerar artefatos que dificultassem a realização da análise.

No total foram utilizadas 81 imagens tomográficas totalizando 133 primeiros molares inferiores. A avaliação das imagens para identificação do canal médio-mesial foi realizada usando os seguintes parâmetros: corte axial mandibular, 6,1 13 segundos de aquisição aos raios X, e tamanho de voxel de $85 \mu \mathrm{m}-200 \mu \mathrm{m}$. O campo de visão variou de $61 \mathrm{~mm}$ x $41 \mathrm{~mm}$ à $61 \mathrm{~mm} \times 78 \mathrm{~mm}$.

Antes da análise das imagens foi realizado um treinamento do examinador responsável, juntamente com um radiologista e um endodontista experiente, que definiram os parâmetros de avaliação das imagens tomográficas. Não foi realizado nenhum teste estatístico de calibração, mas uma série de imagens foram avaliadas e discutidas pelos três profissionais até chegarem em um consenso sobre a análise.

O examinador avaliou as imagens tomográficas em cortes axiais, analisando a anatomia dos canais do terço coronal até o terço apical, procurando identificar a presença ou não do canal médio-mesial, e sua configuração anatômica de acordo com as classificações de Vertucci (1984), Pomeranz et al., (1981), Versiani et al. (2016).

Os dados foram registrados e posteriormente submetidos a uma análise descritiva percentual e análise estatística inferencial em algumas variáveis.

\section{Resultados}

Um total de 81 imagens tomográficas foram analisadas. A partir disso, foram investigados 70 primeiros molares inferiores do lado esquerdo e 63 primeiros molares inferiores do lado direito (Quadro 1). O canal médio-mesial foi identificado em 30 dentes, 23,30\% dos dentes avaliados (Figura 1), sendo 53,33\% deles em dentes do lado esquerdo e 46,66\% no lado direito. Essa diferença não foi estatisticamente significativa pelo teste qui-quadrado ( $\mathrm{p}=0.93$ ).

Nos casos em que foi possível avaliar os primeiros molares inferiores em ambos os hemiarcos de um mesmo paciente ( $\mathrm{n}=52$ / 64,19\%), a presença bilateral do canal médio-mesial não foi observada em nenhum dos casos.

Em relação ao gênero, a incidência foi de 20,63\% (13 dentes) em homens e 24,28\% (17 dentes) em mulheres, diferença de valores que não foi estatisticamente significantes, conforme o teste qui-quadrado ( $\mathrm{P}=0.77)$.

Segundo a análise das imagens, conforme classificação de Vertucci (1984), constatou-se que em 33,3\% (n=10) dos casos o canal médio-mesial apresentava forame independente, classificado como tipo VI ( 1 dente) e VIII ( 9 dentes), enquanto 66,6\% $(n=20)$ dos casos apresentava convergência para um dos canais mesiais (MV ou ML) terminando em um único forame apical, classificação tipo II (Quadro 1).

Segundo classificação de Pomeranz et al., (1981), conforme independência do canal até o forame ou confluência para os canais MV ou ML. A análise das imagens demonstrou que 33,3\% (10 dentes) eram canais independentes até o forame; 46,6\% (14 dentes) confluentes para o canal MV; e 20\% (6 dentes), confluentes para o canal ML (Quadro 1). 
Quadro 1 - Incidência (\%) do canal MM em primeiros molares inferiores por gênero, hemiarco, bilateralidade e classificações de Vertucci [18], Pomeranz et al. [12] e Versiani et al. [17].

\begin{tabular}{|c|c|c|c|}
\hline CATEGORIZAÇÕES & \multicolumn{3}{|c|}{133 PRIMEIROS MOLARES INFERIORES } \\
\hline \multirow{2}{*}{$\begin{array}{l}\text { Quantidade de canais } \\
\text { na raiz mesial }\end{array}$} & 2 canais & 3 canais & 4 canais \\
\hline & $76,70 \%$ & $22,55 \%$ & $0,75 \%$ \\
\hline \multicolumn{2}{|c|}{ MASCULINO } & & \\
\hline Genero & \multicolumn{2}{|l|}{$24,28 \%$} & $\frac{\text { ASCULINO }}{20,63 \%}$ \\
\hline \multirow{2}{*}{ Hemiarco } & \multicolumn{2}{|l|}{ DIREITO } & ESQUERDO \\
\hline & \multicolumn{2}{|l|}{$46,6 \%$} & $53,4 \%$ \\
\hline \multirow{2}{*}{ Localização no arco } & \multicolumn{2}{|c|}{ BILATERAL } & UNILATERAL \\
\hline & \multicolumn{2}{|c|}{0} & $100 \%$ \\
\hline \multirow{2}{*}{ Vertucci (1984) } & II & VI & VIII \\
\hline & $66,6 \%$ & $3,33 \%$ & $30 \%$ \\
\hline \multirow{2}{*}{ Pomeranz et al. (1981) } & CONFLUENTE ML & INDEPENDENTE & CONFLUENTE MB \\
\hline & $20 \%$ & $33,3 \%$ & $46,6 \%$ \\
\hline \multirow{2}{*}{ Versiani et al. (2016) } & INDEPENDENTE & CONFLUENTE & DUPLO CANAL MM \\
\hline & $30 \%$ & $66,6 \%$ & $3,3 \%$ \\
\hline
\end{tabular}

Fonte: Autores.

Figura 1. Imagens axiais de tomografias apresentando 3 e 4 canais na raiz mesial.

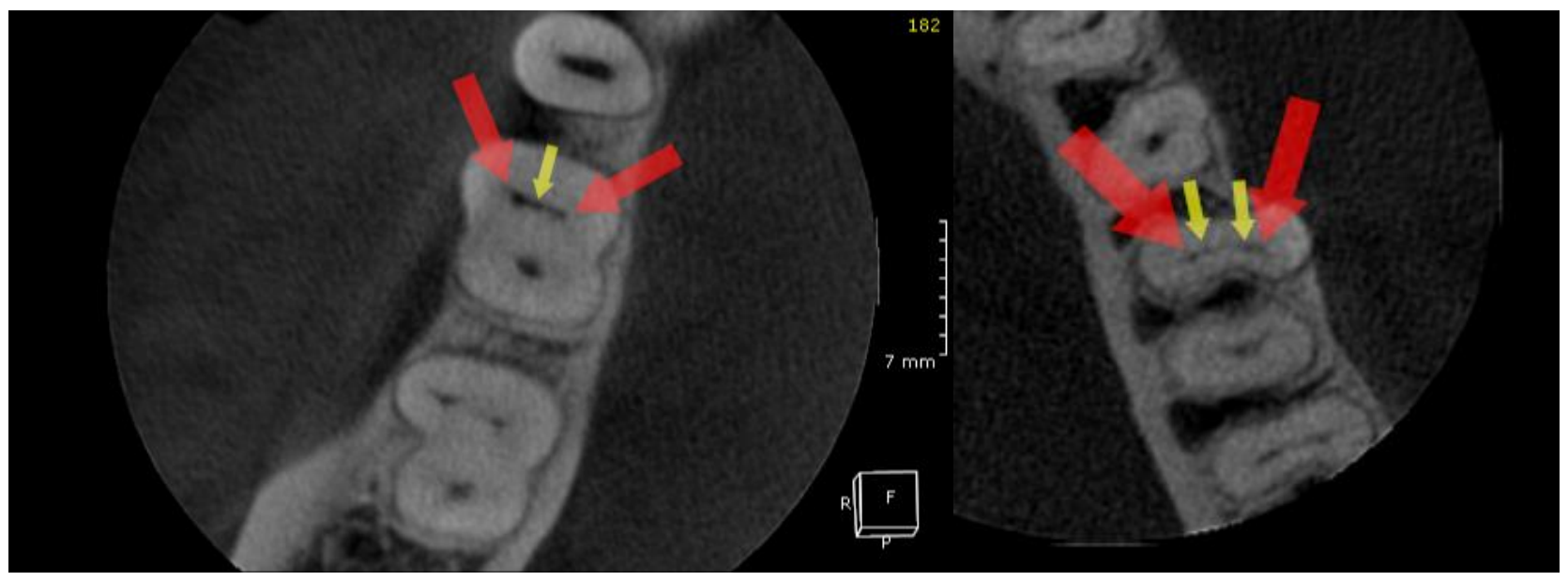

As setas vermelhas identificam os canais MV e ML; as setas amarelas, o(s) canal(ais) MM. Fonte: Autores.

\section{Discussão}

A amostra utilizada contou com todo o banco de dados disponível na clínica radiológica, dentro dos critérios de inclusão, sendo que 133 dentes foram avaliados. Dentre os estudos revisados na literatura, a quantidade da amostra utilizada variou entre 24 à 143 dentes (Karapinar-Kazandag et al., 2010; de Toubes et al., 2012; Nosrat et al., 2015; Chavda et al., 2016; Tahmasbi et al., 2017; Srivastava et al., 2018; Honap et al., 2020; Kuzekanani et al., 2020; Weinberg et al, 2020) e poucos trabalhos utilizaram amostra representativamente superior como Huang et al. (2010), Versiani et al. (2016), Xu et al. (2020) e Yang et al. (2020), com amostras de 237, 258, 357 e 1750 dentes, respectivamente.

Uma vez que foram utilizadas todas as imagens de primeiros molares inferiores disponíveis no banco de dados da clínica radiológica, a variação do tamanho do campo de visão da imagem (FOV - Field ofview) no aparelho tomográfico foi decorrente da solicitação e indicação do exame tomográfico. FOV maior foi utilizado quando não havia necessidade de alta 
resolução nos exames, e menor FOV, para casos que necessitavam maior precisão, haja vista nem sempre a indicação do exame tenha sido para diagnóstico endodôntico.

A importância da TCFC na endodontia, especialmente para a localização e estabelecimento da anatomia do canal MM em primeiros molares inferiores, está demonstrada nos resultados apresentados. Isso também está demonstrado em inúmeros estudos revisados (La et al., 2010; de Toubes et al., 2012; Chavda \& Garg, 2016; Versiani et al., 2016; Tahmasbi et al., 2017; Srivastava et al., 2018; Tomaszewska et al., 2018; Honap et al., 2020; Kuzekanani et al., 2020; Weinberg et al., 2020; Xu et al., 2020; Yang et al., 2020) que utilizaram a TCFC como critério de avaliação isoladamente, ou em comparação com outros métodos.

No presente estudo o canal MM foi localizado em 23,30\% da amostra total dos dentes avaliados por meio das tomografias, sendo possível destacar, na literatura revisada, diferentes resultados nas mais variadas metodologias, que variaram entre zero (Huang et al., 2010) e 45,85\% (Chavda et al., 2016). Dentre as metodologias utilizadas destacam-se o uso da tomografia (Huang et al., 2010 - 0\%; Tahmasbi et al., 2017 - 26\%; Srivastava et al., 2018 - 18,2\%; Kuzekanani et al., 2020 8,1\%; Weinberg et al, 2020; Xu et al., 2020 -3,1\%; e, Yang et al.,2020 - 9,03\%), tomografia associada à magnificação (de Toubes et al., 2012 - 34\%; Chavda et al., 2016 - 45,8\%; e, Honap et al., 2020 - 18,33\%), dentre outras metodologias (Karapinar-Kazandag et al., 2010 - 18\%; Nosrat et al., 2015 - 22\%; e, Versiani et al., 2016-18,6\%). Essa grande variedade de achados pode ser atribuída, além das metodologias utilizadas, à discrepância nas amostras utilizadas, quantidade de casos clínicos realizados, diferentes etnias, dentes tratados e quantidade de participantes nos diferentes estudos.

Quanto à presença do canal $\mathrm{MM}$ em ambos os primeiros molares inferiores dos pacientes, nesse estudo, nos casos em que foi possível avaliar ambos os molares, não foi detectado nenhum caso de bilateralidade. Somente o estudo de Yang et al. (2020) avaliaram essa categoria e relataram um achado de 5,7\%.

Em relação ao gênero, nesse estudo, não houve diferença estatisticamente significante na incidência entre mulheres e homens o que também foi constatado em outros estudos (Huang et al., 2010; Nosrat et al., 2015; Tahmasbi et al., 2017; Srivastava et al., 2018; Xu et al., 2020; Yang et al., 2020).

Nesse estudo, o canal MM era independente até o forame em 33,3\% dos casos. Outros valores foram constatados nos estudos de Chavda et al., (2016) - 9,09\%, Versiani et al. (2016), 6,3\%, Tahmasbi et al. (2017) - 15\%, Srivastava, Alrogaibah \& Aljarbou (2018) - 53,84\%, Xu et al. (2020) - 27,27\%, bem como Weinberg et al. (2020), em que foi identificado em somente um caso ou Karapinar-Kazandag et al., (2010), nenhum caso. A incidência mais frequente, com exceção dos resultados de Srivastava et al., (2018), foi a confluência do canal MM para um dos canais MV ou ML, resultando em um único forame apical (Nosrat et al., 2015; Chavda et al., 2016, Versiani et al., 2016; Tahmasbi et al., 2017; Srivastava et al., 2018; Weinberg et al., 2020; Xu et al., 2020).

Quando classificados segundo Vertucci (1984), no presente estudo, os maiores achados foram para o tipo II (66,6\%), tipo VIII (30\%) e o tipo III (3,3\%). Assim como nesse estudo, Aminshobani et al. (2010) e Yang et al. (2020) também obtiveram maior prevalência na classificação tipo II, respectivamente $66,6 \%$ e 53,8\%. O estudo de Versiani et al. (2016), por meio de microtomografia computadorizada, conseguiu identificar, de forma nítida, a real configuração do sistema de canais radiculares da raiz mesial dos primeiros molares inferiores, e realizaram a seguinte classificação conforme configuração anatômica quando presente o canal médio-mesial: 3 canais independentes; confluência com outro canal, com ou sem istmo entre os canais; e, presença de dois canais médio-mesiais com forames independentes. No estudo de Versiani et al. (2016), assim como no presente estudo, somente 1 caso de quatro canais independentes foram identificados, incidência de $0,38 \%$ e $0,75 \%$, respectivamente.

Com base no que foi exposto, destaca-se a importância da tomografia computadorizada de feixe cônico para a avaliação do sistema de canais dos dentes a serem submetidos ao tratamento endodôntico. Além disso, é necessário que o 
profissional tenha o conhecimento da morfologia do dente a ser tratado e os recursos de imagem auxiliares para um correto diagnóstico e condução clínica nos casos de grande e complexa variação anatômica.

\section{Conclusão}

De acordo com os dados obtidos observou-se a localização e frequência do canal MM em 23,30\% dos primeiros molares inferiores.

\section{Referências}

Aminsobhani, M., Bolhari, B., Shokouhinejad, N., Ghorbanzadeh, A., Ghabraei, S., \& Rahmani, M. B. (2010). Mandibular first and second molars with three mesial canals: a case series. Iranian Endodotic Journal., 5(1), 36-39. https://doi.org/10.22037/IEJ/V5I1.1605.

Chavda, S. M., \& Garg, S. A. (2016). Advanced methods for identification of middle mesial canal in mandibular molars: An in vitro study. Endodontology, 28(2), 92-96. https://doi.org/10.4103/0970-7212.195425.

Cotton, T. P., Geisler, T. M., Holden, D. T., Schwartz, S. A., \& Schindler, W. G. (2007). Endodontic applications of cone-beam volumetric tomography. Journal of Endodontics, 33(9), 1121-32. https://doi.org/10.1016/j.joen.2007.06.011.

Honap, M. N., Devadiga, D., \& Hedge, M. N. (2020). To assess the occurrence of middle mesial canal using cone-beam computed tomography and dental operating microscope: An in vitro study. Journal of Conservative Dentistry, 23(1), 51-56. https://doi.org/10.4103/JCD.JCD_462_19.

Huang, C.C., Chang, Y. C., Chuang, M. C., Lai, T. M., Lai, J. Y., Lee, B. S., \& Lin, C. P. (2010). Evaluation of root and canal systems of mandibular first molars in Taiwanese individuals using cone beam computed tomography. Journal of the Formosan Medical Association, 109(4), 303-308. https://doi.org/ 10.1016/S0929-6646(10)60056-3.

Karapinar-Kazandag, M., Basrani, B. R., \& Friedman, S. (2010). The operating microscope enhances detection and negotiaion of accessory mesial canals in mandibular molars. Journal of Endodontics, 36(8), 1289-94. https://doi.org/10.1016/j.joen.2010.04.005.

Kuzekanani, M., Walsh, L. J., \& Amiri, M. (2020). Prevalence and distribution of the middle mesial canal in mandibular first molar teeth of the Kerman population: A CBCT study. Int J Dent., 1-6. https://doi.org/10.1155/2020/8851984.eCollection2020.

La, S. H., Jung, D. H., Kim, E. C., \& Min, K. S. (2010). Identification of independent middle mesial canal in mandibular first molar using cone-beam computed tomography imaging. Journal of Endodontics, 36(3), 542-5. https://doi.org/10.1016/j.joen.2009.11.008.

Nosrat, A., Deschenes, R. J., Tordik, P. A., Hicks, M. L., \& Fouad, A. F. (2015). Middle Mesial Canals in Mandibular Molars: Incidence and Related Factors. Journal of endodontics, 41(1), 28-32. https://doi.org/10.1016/j.joen.2014.08.004.

Patel, S., Dawood, A., Ford, T. P., \& Whaites, E. (2007). The potential applications of cone beam computed tomography in the management of endodontic problems. International Endodontic Journal, 40(10), 818-30. https://doi.org/10.1111/j.1365-2591.2007.01299.x.

Patel, S., Brown, J., Semper, M., Abella, F., \& Mannocci, F. (2019). European Society of Endodontology position statement: Use of cone beam computed tomography in Endodontics. International Endodontic Journal, 52(12), 1675-78. https://doi.org/10.1111/iej.13187.

Pomeranz, H. H., Eidelman, D. L., \& Goldberg, M. G. (1981). Treatment considerations of the middle mesial canal of mandibular first and second molars. $J$ Endod., 7(12), 565-8. https://doi.org/10.1016/S0099-2399(81)80216-6.

de Toubes, K. M. P. S., Côrtes, M. I. S., Valadares, M. A. A., Fonseca, L. C, Nunes, E., \& Silveira, F. F. (2012). Comparative analysis of accessory mesial canal identification in mandibular first molars by using four different diagnostic methods. Journal of Endodontics, 38(4), 436-441. https://doi.org/10.1016/j.joen.2011.12.035.

Srivastava, S., Alrogaibah, N. A., \& Aljarbou, G. (2018). Cone-beam computed tomographic analysis of middle mesial canals and isthmus in mesial roots of mandibular first molars-prevalence and related factors. Journal of Conservative Dentistry, 21(5), 526-30. https://doi.org/10.4103/JCD.JCD_205_18.

Tahmasbi, M., Jalali, P., Nair, M. K., Barghan, S., \& Nair, U. P. (2017). Prevalence of Middle Mesial Canals and Isthmi in the Mesial Root of Mandibular Molars: an In Vivo Cone-beam Computed Tomographic Study. Journal of Endodontics, 43(7), 1080-3. https://doi:10.1016/j.joen.2017.02.008.

Tomaszewska, M. I., Skinningsrud, B., Jarzebska, A., Pekala, J. R., Tarasiuk, J., \& Iwanaga, J. (2018). Internal and External Morphology of Mandibular Molars: An Original Micro-CT Study and Meta-Analysis with Review of Implications for Endodontic Therapy. Clinical Anatomy, 31(6), 797-811. https://doi.org/10.1002/ca.23080.

Versiani, M., Zapata, R. O., Keles, A., Alcin, H., Bramante, C. M., Pécora, J. D., \& Souza-Neto, M. D. (2016). Middle mesial canals in mandibular first molars: A micro-CT study in different populations. Archives of Oral Biology, 61, 130-137. https://doi.org/10.1016/j.archoralbio.2015.10.020.

Vertucci, J. F. (1984). Root canal anatomy of the human permanent teeth. Oral Surgery, Oral Medicine and Oral Pathology, 58(5), 589-99. https://doi.org/10.1016/0030-4220(84)90085-9.

Weinberg, E. M., Pereda, A. E., Khurana, S., Lotlikar, P. P., Falcon, C., \& Hirschberg, C. (2020). Incidence of Middle Mesial Canals Based on Distance between Mesial Canal Orifices in Mandibular Molars: A Clinical and Cone-beam Computed Tomographic Analysis. Journal of Endodontics, 46(1), 40-43. https://doi.org/10.1016/j.joen.2019.10.017. 
Research, Society and Development, v. 11, n. 3, e50711326822, 2022

(CC BY 4.0) | ISSN 2525-3409 | DOI: http://dx.doi.org/10.33448/rsd-v11i3.26822

Xu, S., Dao, J., Liu, Z., Zhang, Z., Lu, Y.U., \& Zeng, X. (2020). Cone-beam computed tomography investigation of middle mesial canals and isthmuses in mandibular first molars in a Chinese population. BMC Oral Health, 20, 135. https://doi.org/10.1186/s12903-020-01126-2.

Yang, Y., Wu, B., Zeng, J., \& Chen, M. (2020). Classification and morphology of middle mesial canals of mandibular first molars in a Southern chinesesubpopulatio: a cone-beam computed tomographic study. BMC Oral Health., 20(1), 358. https://doi.org/10.1186/s12903-020-01339-5. 